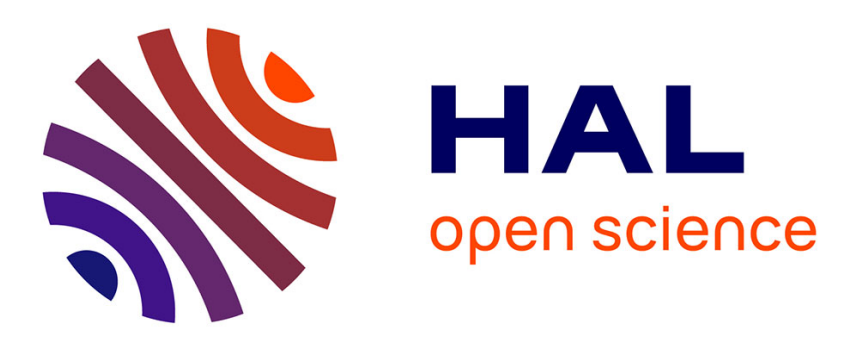

\title{
UPWM-based Pulse Position Modulation for Optical Camera Communications
}

Pengfei Luo, Tong Jiang, Paul Anthony Haigh, Zabih Ghassemlooy, Stanislav

\author{
Zvanovec
}

\section{- To cite this version:}

Pengfei Luo, Tong Jiang, Paul Anthony Haigh, Zabih Ghassemlooy, Stanislav Zvanovec. UPWM-based Pulse Position Modulation for Optical Camera Communications. 2018 11th International Symposium on Communication Systems, Networks and Digital Signal Processing (CSNDSP), Jul 2018, Budapest, Hungary. pp.1-6, 10.1109/CSNDSP.2018.8471874 . hal-02613013

\section{HAL Id: hal-02613013 https://hal.science/hal-02613013}

Submitted on 19 May 2020

HAL is a multi-disciplinary open access archive for the deposit and dissemination of scientific research documents, whether they are published or not. The documents may come from teaching and research institutions in France or abroad, or from public or private research centers.
L'archive ouverte pluridisciplinaire HAL, est destinée au dépôt et à la diffusion de documents scientifiques de niveau recherche, publiés ou non, émanant des établissements d'enseignement et de recherche français ou étrangers, des laboratoires publics ou privés. 


\title{
UPWM-based Pulse Position Modulation for Optical Camera Communications
}

\author{
Pengfei Luo ${ }^{1}$, Tong Jiang ${ }^{1}$, Paul Anthony Haigh ${ }^{2}$, Zabih Ghassemlooy ${ }^{3,3 a}$, Stanislav Zvanovec ${ }^{4}$ \\ ${ }^{1}$ Research Department of HiSilicon, Huawei Technologies Co., Ltd, Beijing, China \\ E-mail: \{oliver.luo, toni.jiang\}@hisilicon.com \\ ${ }^{2}$ Department of Electronic and Electrical Engineering, University College London, London, UK \\ Email: p.haigh@ucl.ac.uk \\ ${ }^{3}$ Optical Communications Research Group, NCRLab, Faculty of Engineering and Environment, \\ Northumbria University, Newcastle-upon-Tyne, UK \\ ${ }^{3 a}$ QIEM, Haixi Institutes, Chinese Academy of Sciences, Quanzhou, China \\ Email: z.ghassemlooy@northumbria.ac.uk \\ ${ }^{4}$ Department of Electromagnetic Field, Faculty of Electrical Engineering, \\ Czech Technical University in Prague, 2 Technicka, 16627 Prague, Czech Republic \\ Email: xzvanove@fel.cvut.cz
}

\begin{abstract}
An undersampled pulse width modulation (UPWM)-based pulse position modulation (UPPM) scheme with the ability to support backward compatibility of UPWM is proposed for the rolling shutter-based optical camera communication systems. An adaptive-modulation scheme and a dedicated segment waveform are also proposed to increase the throughput and to solve several potential problems. Experimental tests are performed to proof the feasibility of the proposed UPPM scheme.
\end{abstract}

Keywords-UPWM; UPPM; modulation; optical camera communication; rolling shutter; undersample

\section{INTRODUCTION}

Optical camera communications (OCC) [1] provide unique opportunities for establishing short-range visible light communication (VLC) links by using a camera as the optical receiver $(\mathrm{Rx})$. Due to the special structure of the camera, there are many advantages of using OCC [2] including: $i$ ) noninterference communications - light incoming from different directions will be received by different pixels; $i$ ) simple signal processing — the Light-emitting diode (LED) pattern which contains useful information can be easily extracted from the whole image, thus complex signal processing is not required; iii) color separation - image sensors (IS) with red, green and blue (RGB) filters are able to receive red, green and blue light simultaneously, and the received RGB signal can therefore be separated simply in digital signal domain.

There are many applications of OCC technology, such as: vehicle-to-vehicle communications for traffic information exchange in order to improve road safety, high accuracy indoor positioning service, or museum guide with reception of geocontextualized multimedia content.

Undersampled-based modulation schemes [3, 4] have been proposed to establish a non-flickering OCC link. However, since the most common frame rates $f_{c}$ of ordinary cameras are less than $100 \mathrm{fps}$ (e.g., 24, 30, 50 and $60 \mathrm{fps}$ ), high-speed communications (e.g., $>1 \mathrm{kbps}$ ) are not easy to be implemented with a single LED. One possible solution is to adopt the rolling shutter mechanism of the complementary metal oxide semiconductor (CMOS)-based IS to record light signal in the form of bright and dark strips from the captured video frames [5]. However, this method might not be valid once the captured light pattern is too small (e.g., in the case of longer range communications) [6]. In this paper, an OCC modulation scheme along with the corresponding symbol structure are proposed to support both rolling shutter-based short-distance high-speed and undersampled-based long-distance low-speed OCC links, so the receivers for [3-5] can be used here directly.

The rest of the paper is organized as follows: Section II introduces the basic principle of the undersampled pulse width modulation (UPWM). In Section III, the UPWM-based pulse position modulation (UPPM) is proposed. The UPPM symbol structure is detailed in Section IV. Experimental results can be found in Section V. Finally, conclusions and future works are given in Section VI.

\section{UNDERSAMPLED PULSE WIDTH MODULATION}

UPWM is proposed to provide a non-flickering OCC link using a low frame rate camera [7]. As illustrated in Fig. 1, this modulation scheme involves two steps. Firstly, a pseudorandom binary stream is mapped to $m$-ary duty cycle (DC) symbols by the $m$-DC mapper module. Secondly, the generated DC symbols are transferred into UPWM symbols with a symbol duration $T_{c}\left(T_{c}=1 / f_{c}\right.$, where $f_{c}=30 \mathrm{fps}$ generally). Each UPWM symbol contains multiple segments of the pulse width modulation (PWM) waveforms, see Fig. 1(d), and odd and even indexed segments are labeled as $W_{1}$ and $W_{2}$, respectively. Note that, $W_{1}$ and $W_{2}$ share the same PWM fundamental frequency $f_{p}=1 / T_{p}$ and the same time duration $T_{s}$ (where $T_{s}=T_{c} /(2 k)$ ) and $k$ is the repeating number of $W_{1}$ and $W_{2}$ in a UPWM symbol. The parameter $k$ is suggested to be set to 5 for the frame rate of $30 \mathrm{fps}$, thus resulting in 10 segments of PWM waveforms in each UPWM symbol with a segment duration $T_{s}=1 / 300 \mathrm{~s}$ (note that, in Fig. $1, k$ is set to 2 for a better demonstration). The DCs of all odd indexed segments 
$W_{1}$ are equal to that of the corresponding DC value $D$ (they share the same color as outlined in Fig. 1), while the DCs of all even indexed segments $W_{2}$ are $1-D$, which is the complementary of $D$.

(a)

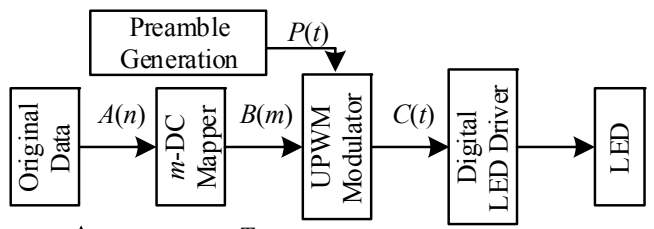

(b)

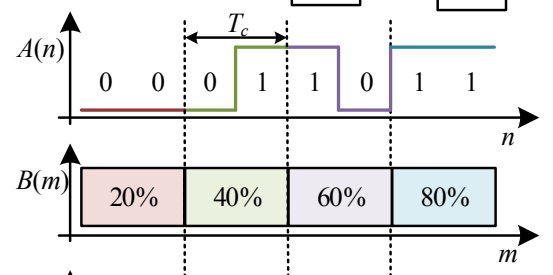

(d)

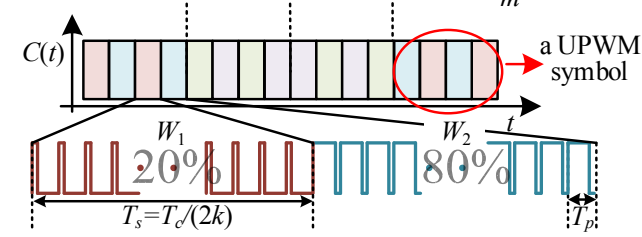

Fig. 1. (a) The block diagram of the transmitter side of a UPWM OCC system, (b) an example of binary data waveform, (c) the corresponding $D$ value to generate a UPWM waveform, and (d) the corresponding UPWM waveform.

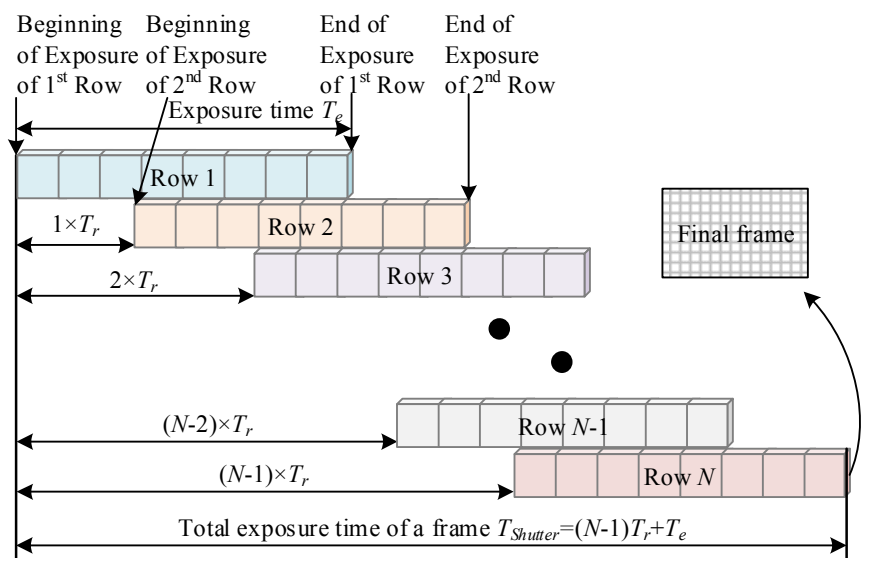

Fig. 2. Diagram demonstrating the frame capturing process of a rolling shutter camera.

Since almost all of the smartphone cameras are rolling shutter-based devices, meaning every row of IS is activated one after another with a fixed line readout delay $T_{r}$ (see Fig. 2, the value of $T_{r}$ normally ranges from 10 to $\left.20 \mu \mathrm{s}\right)$. As we have already known that each segment of the UPWM symbol ( $W_{1}$ or $W_{2}$ ) is composed of numbers of PWM waveforms, and all of those PWM waveforms of the same segment have the same period $T_{p}$ and the same $D$ value. For the UPWM system, the PWM period $T_{p}$ is suggested to be shorter than $T_{e} / 3$, where $T_{e}$ is the exposure time of the receiving camera, to ensure at least three PWM cycles to be integrated during the exposure process. Thus, the stability of the captured brightness can be guaranteed. If we keep decreasing the exposure time $T_{e}$, the cutoff frequency of the IS will be increased. For $T_{e}$ smaller than the shortest PWM pulse width pulse (e.g., $40 \mu \mathrm{s}$ ), the "hidden" pulses of each segment can be captured in the form of a discrete bright-and-dark stripe pattern in each video frame.
Note that, the bright-and-dark strip patterns do not contain any additional information.

\section{UPWM-BASED PULSE Position MOdULATION (UPPM)}

\section{A. Introduction of the UPPM}

In UPWM systems, the PWM $D$ values are used to represent the LED intensity. In order to make the best use of the "hidden" pulse signal in each segment of the UPWM symbol, the pulse position modulation (PPM) signal can be adopted in place of PWM to generate the UPWM symbol. This new modulation scheme is known as UPPM, which could either maintain the backward compatibility with the UPWM Rx or establish a high-speed rolling shutter based UPPM channel.

Fig. 3 compares the traditional UPWM and the proposed UPPM waveforms. It is evident that for a camera with $T_{e}=3 T_{p}$ (here $T_{p}=T_{p p m}$ ) used as a UPWM Rx, the recorded results will (almost) be identical for both transmitted waveforms shown in Fig. 3. However, if $T_{e}<T_{p}$, then bright-and-dark stripe patterns will be captured, and two captured patterns will be definitely different.

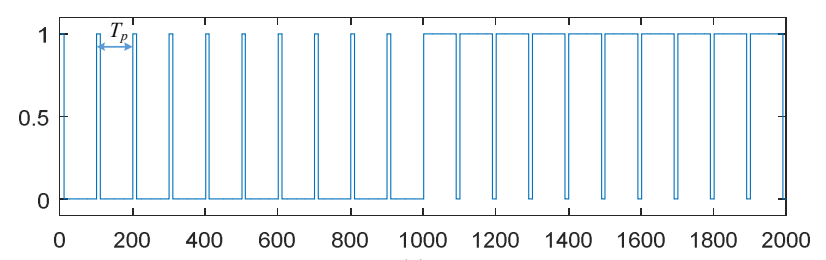

(a)

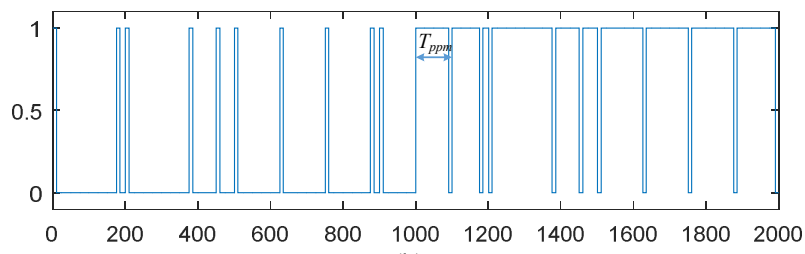

(b)

Fig. 3. Time waveforms with two segments ( $\mathrm{DC}=10 \%, \mathrm{DC}=90 \%$ ). for the: (a) original UPWM, and (b) proposed UPPM.

In order to achieve this backward compatibility with UPWM, the following requirements are necessary to fulfill:

- The embedded PPM symbol period $T_{p p m}$ to be shorter than $T_{e} / 3$.

- All PPM symbols in a certain segment to share the same modulation order.

- The $D$ of each embedded PPM symbol of a hosting segment to be determined by the original UPWM hosting segment (e.g., Fig. 1(c)).

\section{B. MPPM and VMPPM}

In the traditional $M$-ary PPM (MPPM) system, the $n$ bits/symbol are used for each MPPM symbol, where $M$ is the modulation order (i.e., $M=2^{n}$ ). Each MPPM symbol is divided into $M$ time slots, and one square pulse is placed in one of the $M$ adjacent time slots to represent the $n$-bit baseband information data [8].

Table I lists the typical duty cycles $D$ for traditional MPPM symbol for three values of $M$ (i.e., $2,4,8$ ). It is clear that the 
higher the $M$ is, the less power is used. Since, the traditional $M P P M$ symbol has a fixed $D$ for a fixed order $M$, this scheme is not suitable for applications where $D$ variable is subjected. Variable PPM (VPPM) is, therefore, adopted in IEEE 802.15.7 standard [9] to meet the variable $D$ requirements. However, one VPPM symbol only represents one bit, in order to expand the practical use of PPM, variable $M$-ary PPM (VMPPM) scheme is adopted.

\section{TABLE I. TYPICAL DUTY CYCLES OF TRADITIONAL MPPM SYMBOLS AND THE DUTY CYCLE RANGES THAT CAN BE SUPPORTED BY} VMPPM WITH DIFFERENT $M$

\begin{tabular}{|c|c|c|}
\hline $\begin{array}{c}\text { Modulation } \\
\text { order }(\boldsymbol{M})\end{array}$ & $\begin{array}{c}\text { Typical duty } \\
\text { cycle } \boldsymbol{D}\end{array}$ & \begin{tabular}{c} 
Supported duty cycle range \\
\hline 2
\end{tabular} \\
$50 \%$ & $(0 \% \sim 100 \%)$ \\
\hline 4 & $25 \%$ & $(0 \% \sim 25 \%] \&[75 \% \sim 100 \%)$ \\
\hline 8 & $12.5 \%$ & $(0 \% \sim 12.5 \%] \&[87.5 \% \sim 100 \%)$ \\
\hline
\end{tabular}

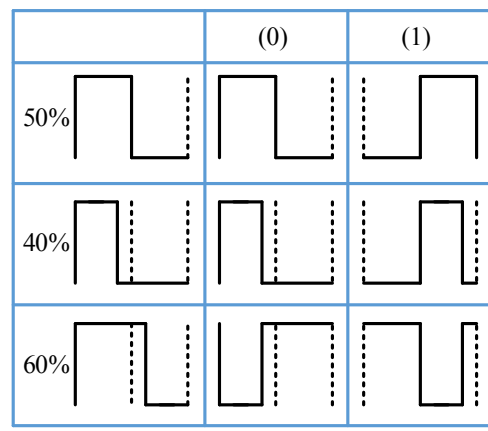

(a)

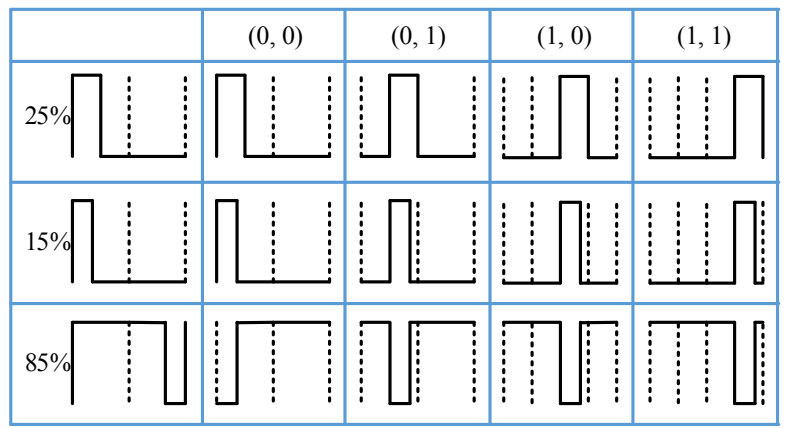

(b)

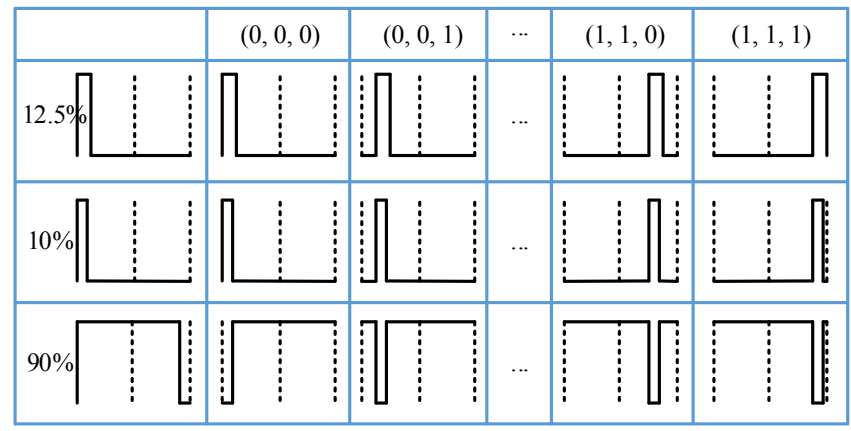

(c)

Fig. 4. Waveforms with different duty cycles of: (a) V2PPM, (b) V4PPM, and (c) V8PPM.

Fig. 4 shows multiple time waveforms with different duty cycles of V2PPM, V4PPM, and V8PPM. Note that, for
V2PPM, if the $D$ is less than $50 \%$, the pulse rising edge is fixed at the beginning of one of the $M$ adjacent time slots, while the pulse width reduced by shifting the falling edge to the left. The same phenomenon can be found when the duty cycles are less than $25 \%$ and $12.5 \%$ for V4PPM and V8PPM, respectively. However, if the $D$ is $>50 \%$ for V2PPM, a V2PPM symbol with a complementary duty cycle of $1-D(1-D<50 \%)$ is firstly generated, then the generated V2PPM with duty cycle of $1-D$ is flipped upside down to obtain the final V2PPM symbol with a $D>50 \%$. Similar symbol generation rules can be found, when the duty cycles are $>75 \%$ and $87.5 \%$ for V4PPM and V8PPM, respectively.

\section{Adaptive-modulation scheme for UPPM}

Table I also lists the duty cycle $D^{\prime}$ ranges, which can be supported by different VMPPM schemes. As shown in the Table I, the $D$ ' range of V2PPM is widest, whereas V8PPM has the narrowest supporting range. Since each V2PPM, V4PPM and V8PPM symbol could carry one, two and three bits, respectively, an adaptive-modulation scheme is therefore proposed for UPPM to maximize the throughput for variable duty cycle $D^{\prime}$.

Because V8PPM could achieve the highest data rate with a fixed symbol duration compared to V4PPM and V2PPM, in addition, V8PPM could only be applied if $D^{\prime}$ is within the range of $(0 \% \sim 12.5 \%]$ and $[87.5 \% \sim 100 \%)$. Once the $D^{\prime}$ of the hosting segment is within this range, V8PPM will be adopted. Similarly, V4PPM and V2PPM can be adopted when $D^{\prime}$ of the hosting segment is within the range of $(12.5 \% \sim 25 \%]$ and $[75 \% \sim 87.5 \%)$ and the range of $(25 \% \sim 75 \%)$, respectively. Table II shows the suggested modulation scheme for a range of $D^{\prime}$.

TABLE II. ADAPTIVE-MODULATION SCHEME

\begin{tabular}{|c|c|}
\hline Duty cycle range & Modulation scheme \\
\hline$(25 \% \sim 75 \%)$ & V2PPM \\
\hline$(12.5 \% \sim 25 \%] \&[75 \% \sim 87.5 \%)$ & V4PPM \\
\hline$(0 \% \sim 12.5 \%] \&[87.5 \% \sim 100 \%)$ & V8PPM \\
\hline
\end{tabular}

\section{Other issues}

Although we suggested that $T_{p p m}<T_{e} / 3$, where $T_{e}$ has normally a low value (e.g., $1 \mathrm{~ms}$ ). Considering all the abovementioned factors, as well as $T_{e}$ ' for UPPM reception $\left(T_{e}{ }^{\prime}\right.$ is normally within the range of $20 \sim 50 \mu \mathrm{s}$ ), additional requirements should be met by the VMPPM symbol:

- The shortest VMPPM pulse width should be longer than $T_{e}, 2$ to ensure that the bright-and-dark strip pattern is distinguishable.

- $T_{p p m}$ should be kept the same for all $M$, and $T_{s}=N_{p p m} \times T_{p p m}$, where $N_{p p m}$ is an integer, which ensures that there will be integer $\left(N_{p p m}\right)$ VMPPM symbols to form a UPWM segment.

\section{DETAIL OF UPPM SYMBOL STRUCTURE}

Each odd or even indexed segment has an equal time period $T_{s}$. The general structure of the segments is the same, see Fig. 5, where $N \%$ is $D$ for the hosting segment. Each segment is 
composed of three regions: the start delimiter, valid data or padding, and the end delimiter. The start delimiter and the end delimiter are used to specify the boundary of a segment. In addition, the start delimiter also indicates the modulation scheme used in this segment. The region between the start delimiter and the end delimiter is used to convey the valid data (e.g., a complete PHY frame, multiple PHY frames, or a part of a PHY frame). If there is no sufficient data, then padding waveforms are included.

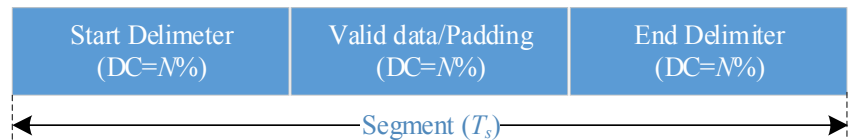

Fig. 5. The general structure of a UPPM symbol.

\section{A. Start delimiter}

Because $D$ of the odd and even indexed segments is complementary, and it keeps changing with the transmitted original data, therefore, at the $\mathrm{Rx}$, different demodulation processes (see Fig. 4 for different modulation schemes) are required in order to successfully recover the transmitted data. A start delimiter and an end delimiter are, therefore, proposed to facilitate the $\mathrm{Rx}$ to distinguish different segments and determine the demodulation scheme.

The start delimiter comprises of two sections, see Fig. 6, the $1^{\text {st }}$ section is used to specify the boundary, while the $2^{\text {nd }}$ section indicates the VMPPM scheme used in this segment.

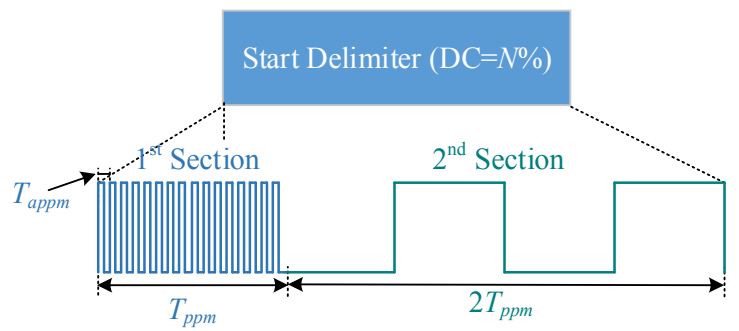

Fig. 6. An example of the structure of start delimiter.

The $1^{\text {st }}$ section represents a period of high speed PWM waveforms with $D$ of $N \%$, which is identical to $D$ of the hosting segment of the UPWM symbol. As shown, this section has a duration of $T_{p p m}$, with the cycle of the high speed PWM pulse $T_{\text {appm }}$ being shorter than $T_{e}, 3$, thus ensuring that a stripe with a brightness of $N \%$ can be captured.

TABLE III. MODULATION ORDER INDICATION.

\begin{tabular}{|c|c|}
\hline Data in the $\mathbf{2}^{\text {nd }}$ section & $\begin{array}{c}\text { Modulation scheme } \\
\text { in the UPPM symbol }\end{array}$ \\
\hline 01 & V2PPM \\
\hline 00 & V4PPM \\
\hline 11 & V8PPM \\
\hline
\end{tabular}

The $2^{\text {nd }}$ section consists of two V2PPM symbols with the same $D$ of $N \%$, and with each V2PPM symbol requiring a duration of $T_{p p m}$. Therefore, three combinations of two V2PPM symbols can be identified by decoding this section. Table III summarizes the modulation schemes and the corresponding data in the $2^{\text {nd }}$ section.

\section{B. Valid data}

The waveforms in the valid data region may carry the PHY frames, which are at first packaged into data groups according to $D$ of the hosting segment $N \%$ and the integer $N_{p p m}$. Then the grouped data is mapped to VMPPM symbols to form the valid data region.

\section{Padding}

If the quantity of data to be transmitted is lower than the capacity of the data region, padding is required. The padding region should comprise of a section of high-speed PWM waveform with a variable duration. The high-speed PWM waveform is the same as the high-speed PWM waveform of the $1^{\text {st }}$ section of the start delimiter.

\section{End delimiter}

The end delimiter is always located at the end of each segment. The waveform in this region is also the same as the waveform in the $1^{\text {st }}$ section of the start delimiter with the same $N \%, T_{p p m}$ and $T_{a p p m}$. Thus, a camera could capture a stripe with a brightness of $N \%$ on receiving this region.

\section{EXPERIMENTAL TESTS AND RESULTS}

In order to test the feasibility of the proposed UPPM scheme as well as the backward compatibility with UPWM, a series of experiments were performed. Since UPPM can be considered as an upgraded UPWM, the same UPWM test-bed shown in Fig. 7 can also be used for UPPM. To ensure each video frame contains sufficient number of bright-and-dark strips, the transmission distance was reduced to $1 \mathrm{~cm}$ when we tested UPPM. Due to the fact that the number of bright-anddark stripes decreases when distance increases, the data rate, therefore, decreases correspondingly. However, in order to maintain the number of bright-and-dark stripes received or to keep the data rate with a longer distance, higher video resolution or telephoto lens (if applicable) are suggested to be used. Thus, if any of the mentioned receiver is employed, the size of LED pattern in the video or the number of bright-anddark stripes will not decrease with an increased distance compared to the OCC system with a lower resolution camera or without a telephoto lens.

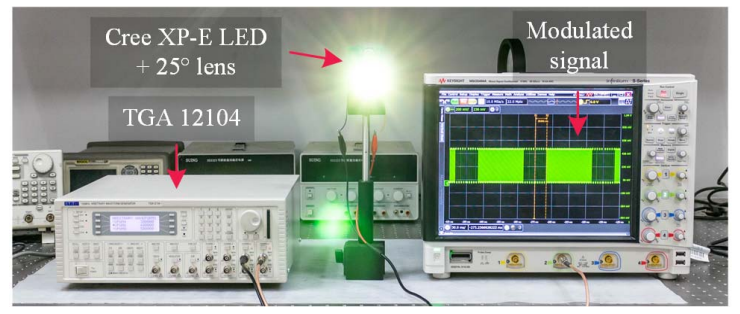

Fig. 7. Tx side of our UPPM OCC experimental test-bed.

At the transmitter (Tx) side, the UPPM signals with unique structures as detailed in Section IV were generated in MATALB and then applied to an arbitrary waveform generator (AWG) (TTi-TGA12104). The output of the AWG was used for intensity modulation of a Cree XP-E LED with a $25^{\circ}$ lens. At the Rx side, a Nexus 6P smartphone (12.3-megapixel Sony Exmor IMX377 sensor with $1.55 \mu \mathrm{m}$ pixels, f/2.0 aperture) 
with an open camera application [10] was used to record the LED, which was continuously transmitting the UPPM signal over the transmission span $d$ of $1 \mathrm{~cm}$. Having captured a short video stream of the $\mathrm{Tx}$, we then carried out the offline processing at the $\mathrm{Rx}$ side.

TABLE IV. KEY PARAMETERS OF EXPERIMENT

\begin{tabular}{|c|c|}
\hline Parameter & Value \\
\hline LED bias & $1.2 \mathrm{~V}$ \\
\hline LED peak to peak voltage & $2 \mathrm{~V}$ \\
\hline Duty cycles $N \%$ & $10 \%, 30 \%, 70 \%, 90 \%$ \\
\hline $\begin{array}{c}\text { VMPPM Modulation } \\
\text { orders } M\end{array}$ & $2,4,8$ \\
\hline $\begin{array}{c}\text { VMPPM symbol duaraion } \\
T_{p p m}\end{array}$ & $1 / 5000 \mathrm{~s}$ \\
\hline UPPM exposure time $T_{e}$ & $\sim 1 / 30000 \mathrm{~s}$ \\
\hline UPWM exposure time $T_{e}$ & $\sim 1 / 1000 \mathrm{~s}$ \\
\hline Segment duration $T_{s}$ & $1 / 300 \mathrm{~s}$ \\
\hline Camera ISO & 60 \\
\hline Video resolution & $1280 \times 720$ \\
\hline
\end{tabular}

For UPPM, the frame rate $f_{c}$ of the camera (Nexus 6p) was set to $30 \mathrm{fps}$. All other key system parameters are shown in Table IV. We carried tests for different modulation orders and for a range of $D$. The captured video frames and the cropped pattern as well as the two segments of transmitted UPPM waveforms are depicted in Fig. 8 to Fig. 10. Note that, the corresponding captured bright-and-dark strip patterns compared to the transmitted waveforms in Fig. 8-10(b) demonstrate the feasibility of the proposed UPPM scheme.

For the UPWM scheme, the camera with the exposure time set to $T_{e}=1 / 1000 \mathrm{~s}$ was placed $1 \mathrm{~m}$ away from the $\mathrm{Tx}$ which was transmitting UPPM with UPWM duty cycles changing from $10 \%$ to $90 \%$. The captured waveform is illustrated in Fig. 11 , which shows that the brightness differences are obvious thus leading to a higher SNR level.

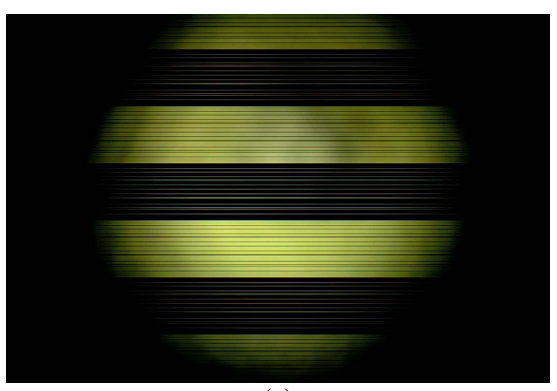

(a)

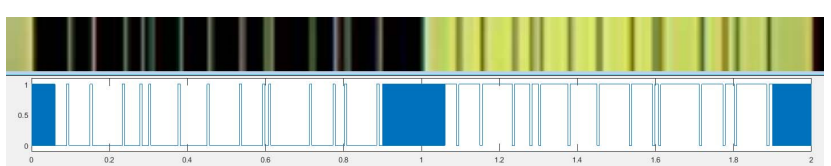

(b)

Fig. 8. (a) The captured bright-and-dark strip pattern for UPPM symbols with V8PPM and $D$ pair of $10 \%$ and $90 \%$, the transmitted V8PPM symbols are $[7,5,0,2,4,7,7,1,7,7,3,6]$; and (b) a comparison of cropped pattern and the transmitted waveform.

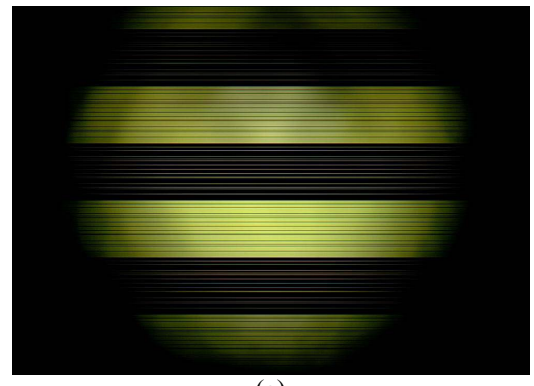

(a)

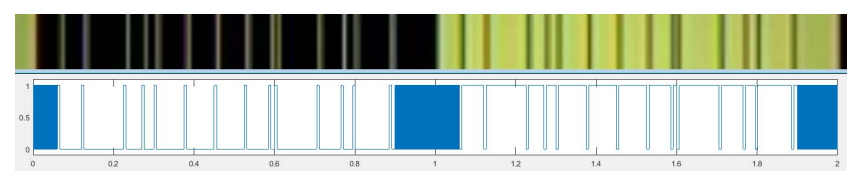

(b)

Fig. 9. (a) The captured bright-and-dark strip pattern for UPPM symbols with V4PPM and $D$ pair of $10 \%$ and $90 \%$, the transmitted V4PPM symbols are $[3,2,0,1,2,3,3,0,3,3,1,3]$; and (b) a comparison of cropped pattern and the transmitted waveform.

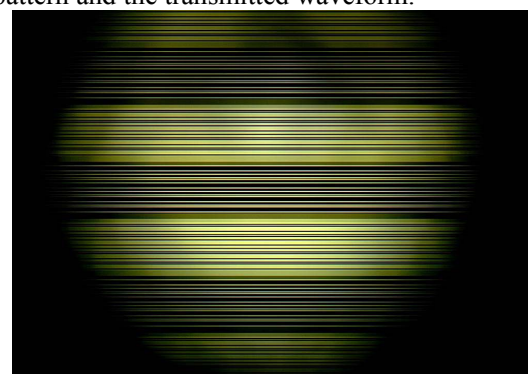

(a)

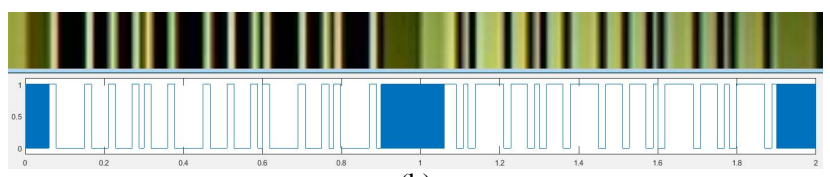

(b)

Fig. 10. (a) The captured bright-and-dark strip pattern for UPPM symbols with V2PPM and $D$ pair of $30 \%$ and $70 \%$, the transmitted V2PPM symbols are $[1,1,0,0,1,1,1,0,1,1,0,1]$; (b) a comparison of cropped pattern and the transmitted waveform.

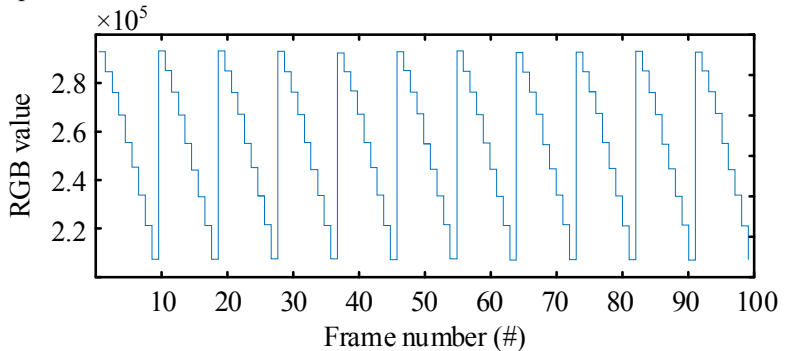

Fig. 11. Captured waveform of the UPPM signal using a camera with an exposure time $T_{e}=1 / 1000 \mathrm{~s}$.

\section{CONCLUSIONS AND FUTURE WORKS}

In this paper, an OCC scheme with UPPM was proposed. UPPM, which is an upgraded version of UPWM, can support both rolling shutter-based short-distance high-speed and undersampled-based long-distance low-speed OCC links. Some specific system requirements and structures for UPPM were given to ensure backward compatibility with UPWM and to allow the $\mathrm{Rx}$ to successfully extract the signal. The 
feasibility of the proposed UPPM scheme was evaluated experimentally.

Future work on this topic will consider the following tasks: i) to find an optimal way to extract signal from the captured bright-and-dark strip patterns; ii) to improve data rate by introducing red, green, and blue lights; iii) to improve data rate by using higher video resolution (e.g., 4K), as well as to find out the relationship among video resolution, data rate and communication distance.

\section{ACKNOWLEDGEMENT}

This work was supported in part by the EPSRC research grant EP/P006280/1: MARVEL and under the Marie Slodowska-Curie ITN grant no. 764461 VisIoN. The Authors would like to acknowledge Professor Min Zhang of Beijing University of Post and Telecommunications for providing experimental resources. P. Luo would like to acknowledge the support from Helen Ouyang.

\section{REFERENCES}

[1] Z. Ghassemlooy, P. Luo, and S. Zvanovec, "Optical Camera Communications," in Optical Wireless Communications: Springer, 2016, pp. 547-568.
[2] N. Serafimovski, V. Jungnickel, Y. M. Jang, and J. Li, "An overview on high speed optical wireless light communications," in "IEEE 802.11 Documents," 2017, Available: https://mentor.iee.org/802.11/dcn/17/1117-0962-02-001c-an-overview-on-high-speed-optical-wireless-lightcommunications.pdf.

[3] P. Luo, Z. Ghassemlooy, H. L. Minh, H.-M. Tsai, and X. Tang, "Undersampled-PAM with subcarrier modulation for camera communications," in OECC, 2015, pp. 1-3.

[4] P. Luo, M. Zhang, Z. Ghassemlooy, S. Zvanovec, S. Feng, and P. Zhang, "Undersampled-based modulation schemes for optical camera communications," IEEE Communications Magazine, vol. 56, no. 2, pp. 204-212, 2018.

[5] C. Danakis, M. Afgani, G. Povey, I. Underwood, and H. Haas, "Using a CMOS camera sensor for visible light communication," in GC Wkshps, 2012, pp. 1244-1248.

[6] T. L. Vu, T. Nguyen, C. S. Kim, E. B. Sin, J. Jeong, and Y. M. Jang, "Survey of indoor optical camera communication (OCC) systems for the Internet of lights," in ICTC, 2017, pp. 700-703.

[7] P. Luo, T. Jiang, P. A. Haigh, Z. Ghassemlooy, and S. Zvanovec, "Undersampled pulse width modulation for optical camera communications," in ICC Workshops, 2018 (accepted).

[8] H. Hemmati, Deep space optical communications. John Wiley \& Sons, 2006.

[9] IEEE Standard for Local and Metropolitan Area Networks--Part 15.7: Short-Range Wireless Optical Communication Using Visible Light, Sept. 62011.

[10] Open Camera. (2018). Open Camera. Available: https://opencamera.sourceforge.io/ 